\title{
Differential expression of SDPR in cancers of the breast.
}

Shahan Mamoor, MS ${ }^{1}$

$3 \quad{ }^{1}$ shahanmamoor@gmail.com

East Islip, NY 11730

Breast cancer affects women at relatively high frequency ${ }^{1}$. We mined published microarray datasets ${ }^{2,3}$ to determine in an unbiased fashion and at the systems level genes most differentially expressed in the primary tumors of patients with breast cancer. We report here significant differential expression of the gene encoding serum deprivation response, SDPR, when comparing primary tumors of the breast to the tissue of origin, the normal breast. SDPR was also differentially expressed in the tumor cells of patients with triple negative breast cancer. SDPR mRNA was present at significantly lower quantities in tumors of the breast as compared to normal breast tissue. Analysis of human survival data revealed that expression of SDPR in primary tumors of the breast was correlated with overall survival in patients with luminal A, basal subtype, and HER2+ cancer, but not in luminal B subtype cancer, demonstrating a relationship between primary tumor expression of a differentially expressed gene and patient survival outcomes influenced by molecular subtype. SDPR may be of relevance to initiation, maintenance or progression of cancers of the female breast.

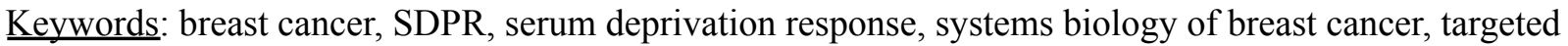
therapeutics in breast cancer. 
Invasive breast cancer is diagnosed in over a quarter of a million women in the United States each year $^{1}$ and in 2018 , breast cancer was the leading cause of cancer death in women worldwide ${ }^{4}$. While patients with localized breast cancer are provided a 99\% 5-year survival rate, patients with regional breast cancer, cancer that has spread to lymph nodes or nearby structures, are provided an $86 \% 5$-year survival rate $^{5,6}$. Patients with metastasis to distant sites, like the brain, are provided a $27 \% 5$-year survival rate ${ }^{5,6}$. Understanding how primary tumors are most transcriptionally different from the tissue from which they originate, the breast, can facilitate development of novel diagnostic and therapeutics to promote early detection and enhanced treatment, and contribute to efforts to prevent progression to metastatic stages. We mined published microarray data ${ }^{2,3}$ to understand at the transcriptome level and in an unbiased fashion genes most differentially expressed in primary tumors of the breast as compared to normal breast tissue. serum deprivation response emerged as among the most differentially expressed genes in cancer of the female breast.

\section{Methods}

We utilized datasets GSE109169² and GSE38959 ${ }^{3}$ for this differential gene expression analysis of female breast cancer. GSE109169 was generated using Affymetrix Human Exon 1.0 ST Array technology with $n=25$ normal breast tissue and $n=25$ tumors of the breast; analysis was performed using platform GPL5175. The tissues from this first dataset are paired tissues (25 tumors matching 25 breast tissues from 25 patients). GSE38959 was generated using Agilent-014850 Whole Human Genome Microarray 4x44K G4112F technology with $n=13$ samples of normal mammary gland ductal cells and $n=30$ samples of tumor cells from patients with triple negative breast cancer; analysis was performed using platform GPL4133. The Benjamini and Hochberg method of p-value adjustment was used for ranking of differential expression but raw $p$-values were used to assess statistical significance of global differential expression. Log-transformation of data was auto-detected, and the NCBI generated category of platform annotation was used. A statistical test was performed to evaluate whether SDPR expression was significantly different between primary breast tumors and breast tissue using a two-tailed t-test. For Kaplan-Meier survival analysis, we used the Kaplan-Meier plotter online tool ${ }^{7}$ for correlation of SDPR mRNA expression levels with overall survival (OS) in $n=404$ patients with basal subtype cancer, $n=794$ patients with luminal A subtype cancer, $n=515$ patients with luminal B subtype cancer, and $n=166$ patients with HER2+ cancer.

\section{Results}

We performed discovery of genes associated with breast cancer in females by mining two independently published microarray datasets ${ }^{2,3}$.

\section{SDPR is differentially expressed in primary tumors of the breast.}

Comparison of 25 normal breast tissues to 25 tumors of the breast ${ }^{2}$ from patients with early-onset breast cancer revealed that the gene encoding serum deprivation response, encoded by the SDPR gene, was among the genes most differentially expressed in tumors of the breast in human breast cancer (Chart 1). When sorting each of the genes expressed in tumors of the breast based on significance of difference as compared to normal breast tissue, SDPR ranked 16 out of 19076 total transcripts, equating to $99.9 \%$ differential expression (Chart 1). Differential expression of SDPR in female breast cancer was statistically significant (Chart $1 ; p=3.27 \mathrm{E}-19)$.

Analysis of a second microarray datase ${ }^{3}$, here in tumor cells of patients with triple negative breast cancer as compared to normal mammary gland ductal cells, again revealed significant differential expression of SDPR in human breast cancer (Chart 2). When sorting each of the genes expressed in the 
tumor cells of patients with triple negative breast cancer based on significance of difference as compared to normal mammary gland ductal cells, SDPR ranked 2678 out of 45015 total transcripts, equating to 94.1\% differential expression (Chart 2). Differential expression of SDPR in the tumor cells of patients with triple negative breast cancer was statistically significant (Chart $2 ; p=1.94 \mathrm{E}-05$ ). These data suggested that differential expression of SDPR was not an artifact of a single microarray dataset, nor was it associated with one type of breast cancer, rather a general feature of cancers of the breast.

\section{SDPR is expressed at significantly lower levels in breast tumors as compared to the breast.}

We obtained exact mRNA expression levels for SDPR from the breast and from breast tumors ${ }^{2}$ to understand the magnitude and direction of SDPR expression change. SDPR was expressed at lower levels in tumors of the breast as compared to normal breast tissue (Figure 1). Decreased expression of SDPR in primary breast tumors was statistically significant (Figure 1: $p<0.0001$ ). SDPR was expressed at $8.15 \pm$ 0.46 arbitrary units (AU) in normal breast tissue, while it was expressed at $6.67 \pm 0.27 \mathrm{AU}$ in tumors of the breast. We calculated a mean fold change of 0.82 in SDPR mRNA levels when comparing primary tumors of the breast to normal breast tissues.

\section{SDPR expression correlates with survival outcomes in luminal A, basal, and HER2+ human breast cancer.}

We performed Kaplan-Meier survival analysis in an attempt to correlate SDPR mRNA expression levels with survival outcomes in patients with breast cancer, evaluating correlations between molecular subtype and overall survival. We observed a statistically significant correlation between SDPR expression and overall survival (OS) in patients with luminal A subtype breast cancer (Figure 2; log rank $p$-value: 0.049 for overall survival, hazard ratio: $0.73(0.53-1)$ (Fig. 2)). SDPR mRNA levels were a positive prognostic indicator in luminal A subtype breast cancer patients. Median OS was 120 months for luminal A patients with low tumor expression of SDPR as compared to luminal A patients with high tumor expression of SDPR, who possessed median OS of 179.01 months (Chart 3). We also observed a statistically significant correlation between SDPR expression and overall survival (OS) in patients with HER2 + subtype breast cancer (Figure 2; $\log$ rank $p$-value: 0.025 for overall survival, hazard ratio: 1.95 (1.08-3.52) (Fig. 2)). SDPR mRNA levels were a negative prognostic indicator in HER2+ subtype breast cancer patients. Median OS was 70.68 months for HER2+ patients with low tumor expression of SDPR as compared to HER2+ patients with high tumor expression of SDPR, who possessed median OS of 34.8 months (Chart 3). We also observed a correlation between SDPR expression and overall survival (OS) in patients with basal subtype breast cancer which trended towards statistical significance (Figure 2; $\log$ rank $p$-value: 0.095 for overall survival, hazard ratio: 1.38 (0.94-2.03) (Fig. 2)). SDPR mRNA levels were a negative prognostic indicator in basal subtype breast cancer patients. Median OS was 75.37 months for basal patients with low tumor expression of SDPR as compared to basal subtype patients with high tumor expression of SDPR, who possessed median OS of 55.46 months (Chart 3). SDPR primary tumor expression was not correlated with overall survival in luminal B subtype breast cancer (Figure 2; $\log$ rank $p$-value: 0.78 for OS, hazard ratio: 0.95 (0.67-1.35) (Fig. 2).

Thus, through comparative transcriptome analysis of primary tumors of the breast and normal breast tissue, we found that differential expression and down-regulation of SDPR was among the most significant transcriptional features in primary tumors from patients with breast cancer. SDPR expression in primary tumors of the breast was correlated with overall survival in patients with luminal A subtype cancers, with SDPR primary tumor mRNA levels a positive prognostic indicator for luminal A patients, but a negative prognostic indicator for basal subtype and HER2+ patients. 


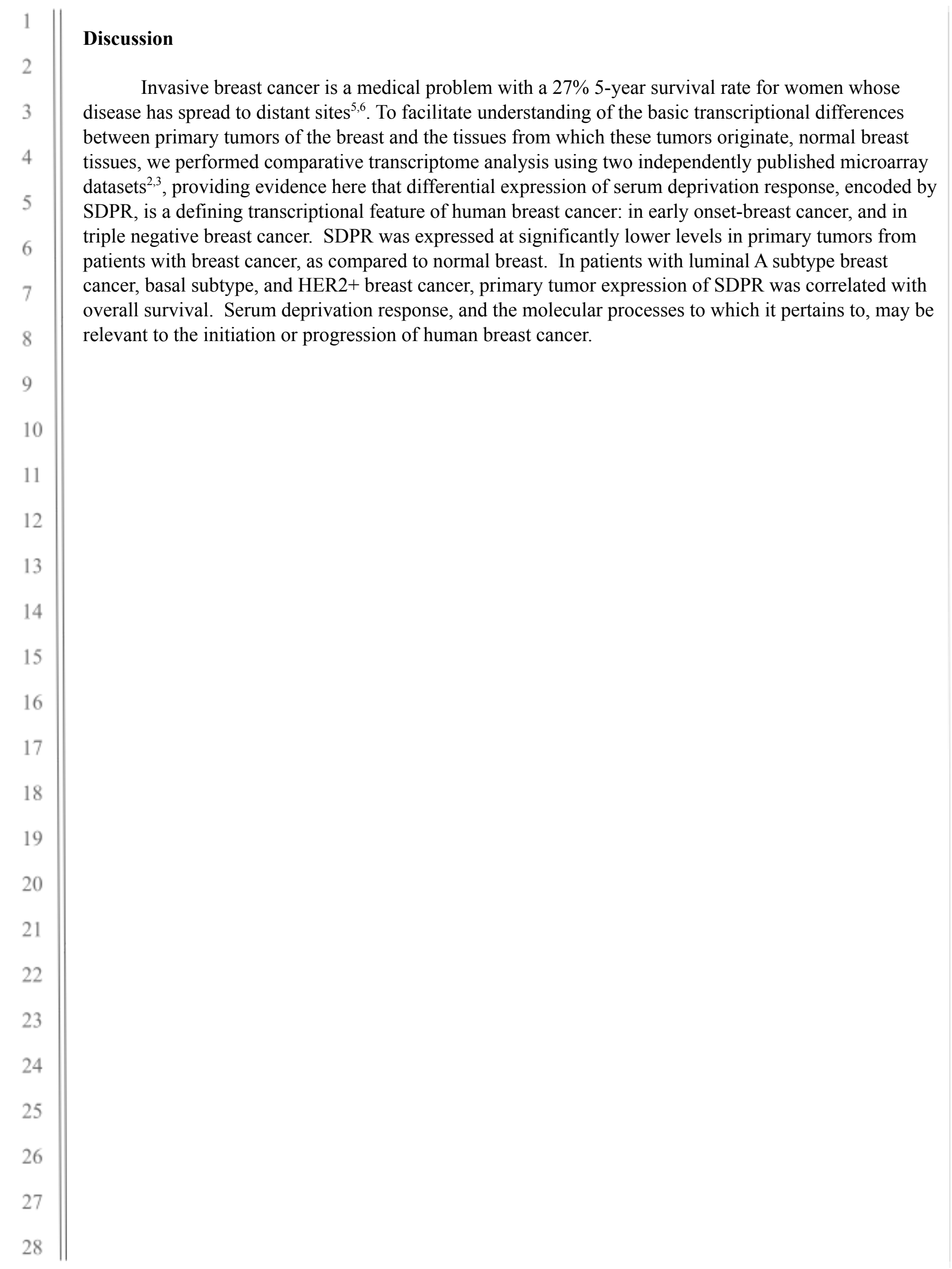




\section{References}

1. DeSantis, C.E., Ma, J., Goding Sauer, A., Newman, L.A. and Jemal, A., 2017. Breast cancer statistics, 2017, racial disparity in mortality by state. CA: a cancer journal for clinicians, 67(6), pp.439-448.

2. Chang, J.W., Kuo, W.H., Lin, C.M., Chen, W.L., Chan, S.H., Chiu, M.F., Chang, I.S., Jiang, S.S., Tsai, F.Y., Chen, C.H. and Huang, P.H., 2018. Wild-type p53 upregulates an early onset breast cancer-associated gene GAS7 to suppress metastasis via GAS7-CYFIP1- mediated signaling pathway. Oncogene, 37(30), pp.4137-4150.

3. Komatsu, M., Yoshimaru, T., Matsuo, T., Kiyotani, K., Miyoshi, Y., Tanahashi, T., Rokutan, K., Yamaguchi, R., Saito, A., Imoto, S. and Miyano, S., 2013. Molecular features of triple negative breast cancer cells by genome-wide gene expression profiling analysis. International journal of oncology, 42(2), pp.478-506.

4. Bray, F., Ferlay, J., Soerjomataram, I., Siegel, R.L., Torre, L.A. and Jemal, A., 2018. Global cancer statistics 2018: GLOBOCAN estimates of incidence and mortality worldwide for 36 cancers in 185 countries. CA: a cancer journal for clinicians, 68(6), pp.394-424.

5. ACS Cancer Facts \& Figures 2019.

https://www.cancer.net/cancer-types/breast-cancermetastatic/statistics.

6. Survival Rates for Breast Cancer. https://www.cancer.org/cancer/breast-cancer/ understanding-a-breast-cancer-diagnosis/breast-cancer-survival-rates.html

7. Györffy, B., Lanczky, A., Eklund, A.C., Denkert, C., Budczies, J., Li, Q. and Szallasi, Z., 2010. An online survival analysis tool to rapidly assess the effect of 22,277 genes on breast cancer prognosis using microarray data of 1,809 patients. Breast cancer research and treatment, 123(3), pp.725-731. 


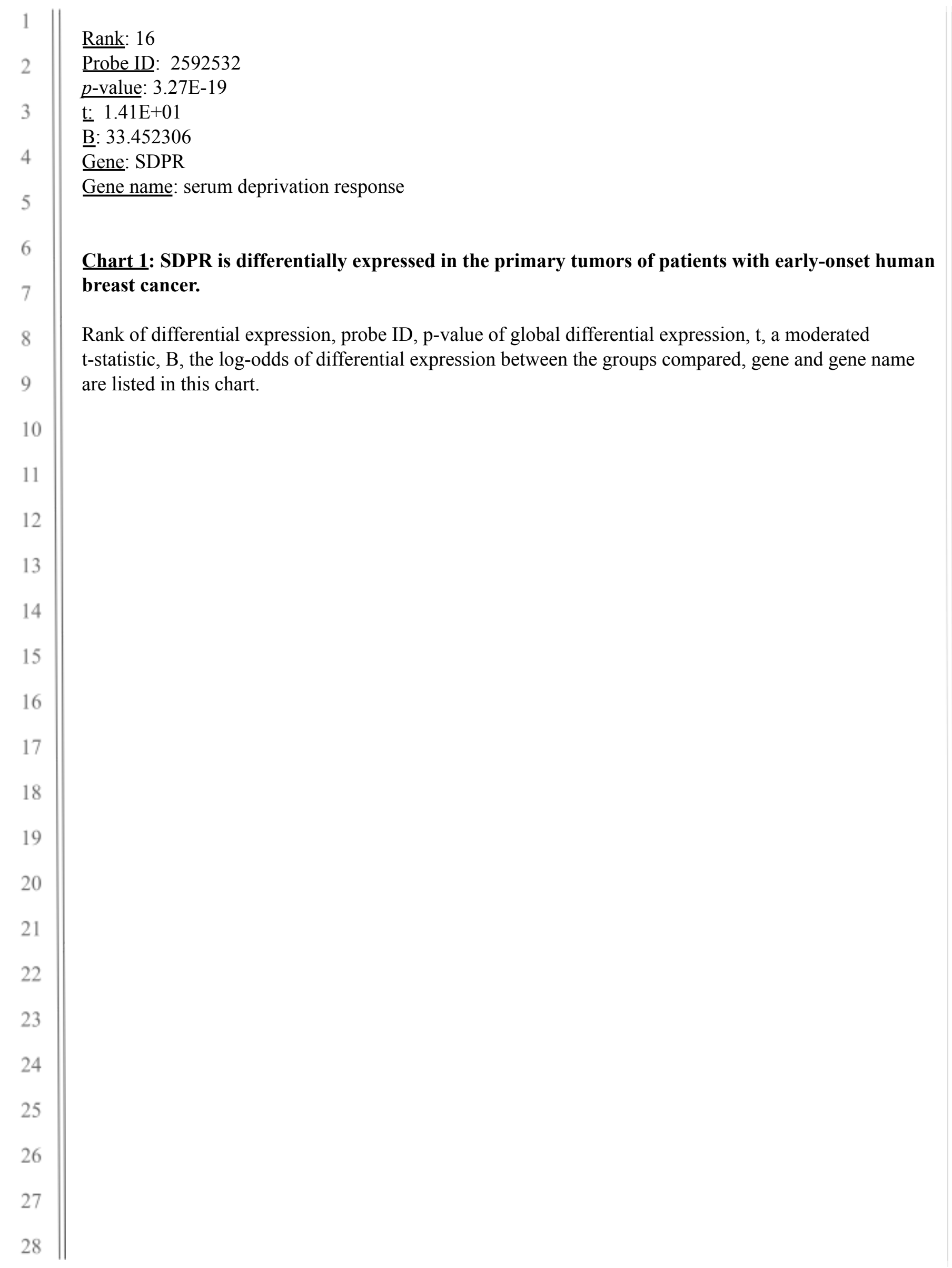




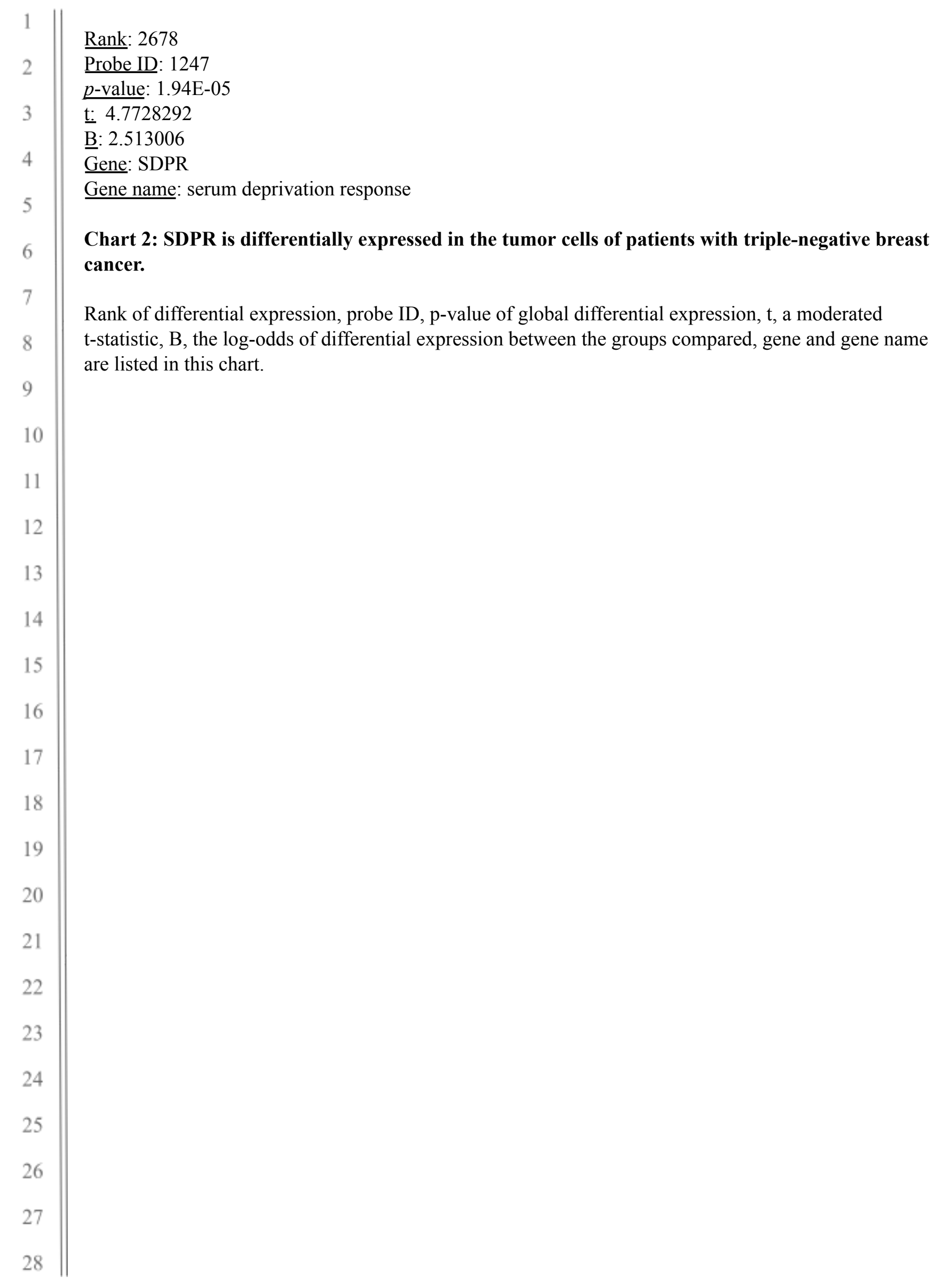




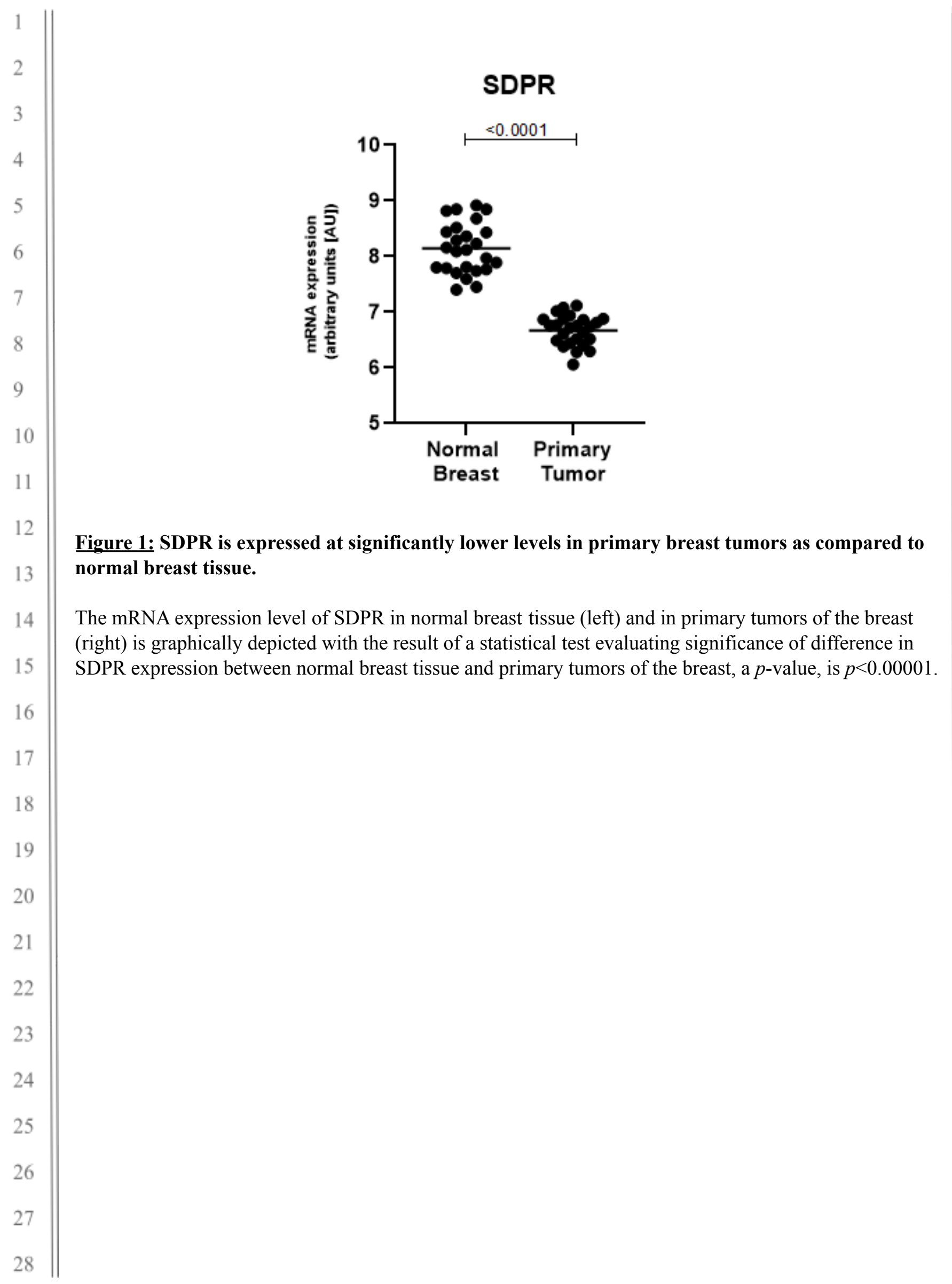









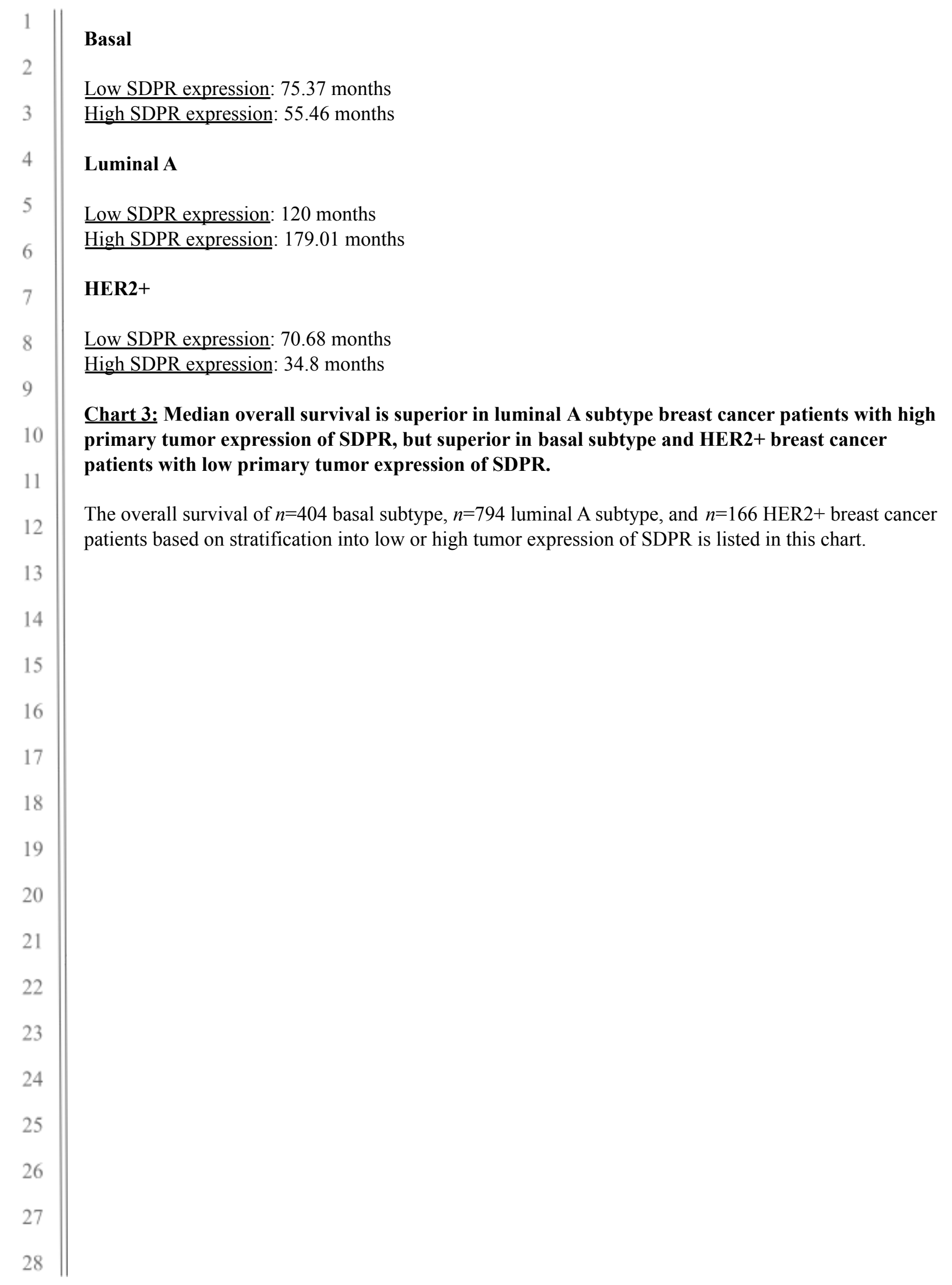

